\title{
Cerebellar-Motor Cortex Connectivity: One or Two Different Networks?
}

\author{
Danny A. Spampinato, ${ }^{1}$ Pablo A. Celnik, ${ }^{2}$ and ${ }^{\circledR}$ John C. Rothwell ${ }^{1}$ \\ ${ }^{1}$ Sobell Department of Motor Neuroscience and Movement Disorders, UCL Queen Square Institute of Neurology, London WC1E 6BT, United \\ Kingdom, and ${ }^{2}$ Department of Physical Medicine and Rehabilitation, Johns Hopkins Medical Institution, Baltimore, Maryland 21205
}

Anterior-posterior (AP) and posterior-anterior (PA) pulses of transcranial magnetic stimulation (TMS) over the primary motor cortex (M1) appear to activate distinct interneuron networks that contribute differently to two varieties of physiological plasticity and motor behaviors (Hamada et al., 2014). The AP network is thought to be more sensitive to online manipulation of cerebellar (CB) activity using transcranial direct current stimulation. Here we probed CB-M1 interactions using cerebellar brain inhibition (CBI) in young healthy female and male individuals. TMS over the cerebellum produced maximal CBI of PA-evoked EMG responses at an interstimulus interval of $5 \mathrm{~ms}$ (PA-CBI), whereas the maximum effect on AP responses was at $7 \mathrm{~ms}$ (AP-CBI), suggesting that $\mathrm{CB}-\mathrm{M} 1$ pathways with different conduction times interact with $\mathrm{AP}$ and $\mathrm{PA}$ networks. In addition, paired associative stimulation using ulnar nerve stimulation and PA TMS pulses over M1, a protocol used in human studies to induce cortical plasticity, reduced PA-CBI but not AP-CBI, indicating that cortical networks process cerebellar inputs in distinct ways. Finally, PA-CBI and AP-CBI were differentially modulated after performing two different types of motor learning tasks that are known to process cerebellar input in different ways. The data presented here are compatible with the idea that applying different TMS currents to the cerebral cortex may reveal cerebellar inputs to both the premotor cortex and M1. Overall, these results suggest that there are two independent CB-M1 networks that contribute uniquely to different motor behaviors.

\section{Significance Statement}

Connections between the cerebellum and primary motor cortex (M1) are essential for performing daily life activities, as damage to these pathways can result in faulty movements. Therefore, developing and understanding novel approaches to probe this pathway are critical to advancing our understanding of the pathophysiology of diseases involving the cerebellum. Here, we show evidence for two distinct cerebellar-cerebral interactions using cerebellar stimulation in combination with directional transcranial magnetic stimulation (TMS) over M1. These distinct cerebellar-cerebral interactions respond differently to physiological plasticity and to distinct motor learning tasks, which suggests they represent separate cerebellar inputs to the premotor cortex and M1. Overall, we show that directional TMS can probe two distinct cerebellar-cerebral pathways that likely contribute to independent processes of learning.

\section{Introduction}

Converging evidence from noninvasive brain stimulation (NIBS) and neuroimaging studies in healthy individuals and in patients with neurologic damage has shown that the cerebellum plays a critical role in optimizing motor control and motor learning

\footnotetext{
Received Oct. 7, 2019; revised Mar. 20, 2020; accepted Mar. 24, 2020.

Author contributions: D.A.S., P.A.C., and J.C.R. designed research; D.A.S. performed research; D.A.S. analyzed data; D.A.S. wrote the paper.

The authors declare no competing financial interests.

D.A.S. and J.C.R. are funded by the Medical Research Council (Grant MR/P006671/1); and P.A.C. is funded by the National Institute of Neurological Disorders and Stroke (Grant 5R01-HD-053793-12). We thank Paul Hammond for technical support, as well as Po-Yu Fong and Kevin Michell for assistance with experiments. Correspondence should be addressed to Danny A. Spampinato at d.spampinato@ucl.ac.uk. https://doi.org/10.1523/JNEUROSCI.2397-19.2020

Copyright $\odot 2020$ the authors
}

by refining motor inhibition (Diedrichsen et al., 2005; Wolpert et al., 2011). The integrity of these actions relies, in part, on cerebellar-thalamic-cortical pathways, as damage to this pathway may result in ataxia or dysmetria (Koziol et al., 2014). These connections have also been implicated in the pathophysiology of dystonia (Argyelan et al., 2009) and other conditions. Thus, gaining further insight into cerebello-cortical connectivity is important if we are to understand the mechanisms that play a role in the pathophysiology of these complex disorders. Developing this understanding could lead to targeting the cerebellum with NIBS, which may improve symptoms associated with disease (Koch et al., 2014; Porcacchia et al., 2019).

In humans, it is possible to investigate cerebello-cortical interactions using the cerebellar brain inhibition (CBI) method. If a conditioning transcranial magnetic stimulation (TMS) pulse 
is given to the lateral cerebellum, corticospinal electromyography (EMG) responses evoked by TMS over contralateral primary motor cortex (M1) are suppressed, specifically when the cerebellar stimuli are given 5-7 ms before M1 stimulation (Ugawa et al., 1995; Pinto and Chen, 2001). The cerebellar TMS is postulated to activate Purkinje cells, which in turn inhibit neurons in the deep cerebellar nuclei, thus reducing the activity of the disynaptic excitatory pathway to M1 (Galea et al., 2009). CBI is sensitive to behaviors where error-based learning mechanisms are required to learn new sensorimotor relationships (Spampinato et al., 2017; Spampinato and Celnik, 2018). Specifically, a release of CBI following learning has been suggested to reflect long-term depression of Purkinje cells (Jayaram et al., 2011) as described in models of motor learning (Ito, 2002). Therefore, changes in CBI may represent the physiological contributions arising from the cerebellum that is associated with error-driven learning.

TMS can also be used to investigate distinct subsets of interneurons within M1. Indeed, changing the direction of the current in M1 can activate independent sets of synaptic inputs to corticospinal neurons, termed I-waves, that reflect indirect depolarization of axons projecting monosynaptically (early Iwaves) and polysynaptically (late I-waves) to output neurons (Delvendahl et al., 2014; Di Lazzaro and Rothwell, 2014). Posterior-to-anterior (PA)-induced currents to the central sulcus consistently recruit early I-waves, whereas anterior-to-posterior (AP)-induced currents activate late I-waves that result in delayed corticospinal activity (Di Lazzaro and Ziemann, 2013). Recent work has shown that excitability changes produced by neurons recruited by AP current directions are more dependent on cerebellar activity than those produced with PA current directions (Hamada et al., 2014). However, these results rely on transcranial direct current stimulation (TDCS) over the cerebellum. This interventional approach has had mixed results in eliciting changes in cerebellar-dependent learning tasks (Jalali et al., 2017) and in triggering consistent changes in cerebellar excitability (Galea et al., 2009).

In the present study, we have therefore used CBI to probe directly the interaction of cerebellum with AP and PA networks in the cerebral cortex. Previous studies have only investigated CBI using PA stimulation over M1. Since responses to PA and AP stimulation of M1 may recruit distinct subpopulations of corticospinal neurons (Witham et al., 2016) and behave differently when probed with intracortical inhibition and sensory afferent inhibition (Hanajima et al., 1998; Hannah and Rothwell, 2017), we first compared the effect of CBI on the response to PA and $\mathrm{AP}$ pulses and found maximal $\mathrm{CBI}$ of $\mathrm{PA}$-evoked responses at an inter-stimulus interval of $5 \mathrm{~ms}$ (PA-CBI) and at $7 \mathrm{~ms}$ for APevoked responses (AP-CBI). In a follow-up experiment, we utilized a plasticity protocol to target PA-inputs rather than APinputs (i.e., repetitive stimulation of PA currents) and found that it had a differential effect on AP-CBI and PA-CBI. The results of both sets of experiments suggested that cerebellar inputs have highly specific connections with different interneuronal pathways in M1. Finally, we show how cerebellar interactions with PA and AP inputs are modulated by two distinct types of motor-learning tasks that process input from the cerebellum in different ways.

\section{Materials and Methods}

\section{Participants}

A total of 38 right-handed, healthy, young volunteers (26 females; mean $\pm \mathrm{SD}$ age, $25.3 \pm 3.7$ years; age range, $18-35$ years) participated in this study. None of the subjects had neurologic disorders or contraindications to TMS, as well as no history of migraines, psychiatric disease, metallic implants, drug abuse, or alcohol abuse (Rossi et al., 2009). Each experiment was conducted in accordance with the Declaration of Helsinki on the use of human subjects. Experiment 1 $(n=12)$ was approved by the Johns Hopkins Medical Institution (JHMI) Institutional Review Board and conducted at JHMI. Experiment $2(n=$ 14) and experiment $3(n=12)$ were conducted at University College London (UCL) and approved by the ethics committee UCL. All participants at each institution were provided written informed consent before participating in the study. The duration of each testing session lasted between 60 and $90 \mathrm{~min}$.

\section{Neurophysiological assessments}

Electromyography recordings. Surface electrodes $(\mathrm{Ag}-\mathrm{AgCl})$ were placed over the first dorsal interosseous (FDI) muscle for recording motorevoked potentials (MEPs) caused by magnetic stimulation. Subjects were instructed to maintain the hand in a relaxed position throughout the entire experiment while EMG activity was monitored. The signal was amplified (gain, 1000), bandpass filtered $(20 \mathrm{~Hz}-3 \mathrm{kHz})$, digitized at a frequency of $2 \mathrm{kHz}$, and stored for offline analysis (catalog \#1401, Cambridge Electronic Design). Data were stored on another computer to complete offline analysis using a variety of custom MATLAB scripts (MathWorks).

Transcranial magnetic stimulation over M1. We stimulated the left M1 using a 70-mm-diameter figure-of-eight TMS coil (200², Magstim) to elicit an MEP of the FDI muscle of the right hand. We used a neuronavigation system (BrainSight, Rogue Research) to ensure the consistency of stimulation over the "hot spot" of the FDI muscle throughout the entire experiment. Previous works have demonstrated that changing the TMS current flow across the hand area of M1 elicits different descending volleys. For instance, applying posterior-anterior-directed current (PA current) preferentially recruits early I-waves, whereas AP current tends to elicit late I-waves (Day et al., 1989; Di Lazzaro et al., $1998,2001)$. In this study, we placed the TMS coil tangential to the scalp to induce either (1) PA-directed currents, with the handle pointed either backward at a $45^{\circ}$ angle to the midline; or (2) AP-directed currents, by placing the coil handle $180^{\circ}$ to the PA currents. For each coil orientation, we also determined the resting motor threshold (rMT), defined as the minimum intensity needed to evoke MEPs of $\geq 50 \mu \mathrm{V}$ in 5 of 10 trials (Rossi et al., 2009). After finding rMT intensity, we determined the stimulator output intensity needed to evoke MEPs of $\sim 1 \mathrm{mV}$ in peak-topeak amplitude.

Cerebellar-M1 connectivity. To assess changes in the connectivity between the cerebellum and M1, we used a well established paired-pulse technique that consists of delivering a TMS conditioned stimulus (CS) over the right cerebellar hemisphere $5 \mathrm{~ms}$ before administering a test stimulus (TS) over the left M1 (Ugawa et al., 1995; Pinto and Chen, 2001; Daskalakis et al., 2004). We stimulated the cerebellum using a 110 $\mathrm{mm}$ double-cone coil (110 mm mean diameter; Magstim). To avoid stimulation of the pyramidal tract with the cerebellar TMS, the intensity for cerebellar stimulation was set at $5 \%$ below the brainstem active MT (BaMT), as described in previous studies (Werhahn et al., 1996; Galea et al., 2009). Specifically, BaMT was tested with a $110-\mathrm{mm}$-diameter double-cone coil centered over the inion with the stimulator current directed downward and was defined as the nearest $5 \%$ stimulator output that elicited an MEP of $50 \mu \mathrm{V}$ in a slightly contracted FDI muscle. If the BaMT was not observed at $75 \%$ of the maximum stimulator output (MSO), then $70 \%$ MSO was used to avoid participant discomfort (Schlerf et al., 2015). Across all studies, we found nine individuals who displayed a BaMT response under 75\% MSO (mean cerebellar conditioning intensity, $68.57 \pm 0.75$; Table 1 ). To assess cerebellar-M1 connectivity (CBI), the double-cone coil was then placed over the right cerebellar cortex $3 \mathrm{~cm}$ lateral to the inion, with the stimulator current directed downward (Hardwick et al., 2014; Ginatempo et al., 2019; Spampinato et al., 2020). Moreover, the TS over left M1 was delivered using a 70-mm-diameter figure-of-eight coil, and the intensity was set to a stimulator output that elicited $\sim 1 \mathrm{mV}$ MEP response. This required the presence of the following two experimenters: one to hold the figure-of-eight coil over M1 guided by neuronavigation; and another individual to hold the 
Table 1. Shown are the mean \pm SEM of M1 coil intensities (columns 1 and 2) and MEP latencies (columns 3 and 4) for each current direction, as well as the cerebellar coil intensities (column 5) used across each experiment

\begin{tabular}{|c|c|c|c|c|c|}
\hline & $\begin{array}{l}\text { PA intensity } \\
\text { (\% MSO) }\end{array}$ & $\begin{array}{l}\text { AP intensity } \\
\text { (\% MSO) }\end{array}$ & PA latency & AP latency & $\begin{array}{c}\text { Cerebellar intensity } \\
(\% \mathrm{MSO})\end{array}$ \\
\hline Experiment 1 & $43.41 \pm 2.57$ & $58.91 \pm 3.02$ & $21.02 \pm 0.34$ & $22.86 \pm 0.41$ & $68.33 \pm 0.90$ \\
\hline Experiment 2 & $46.29 \pm 2.27$ & $60.21 \pm 3.35$ & $21.37 \pm 0.29$ & $22.44 \pm 0.33$ & $67.14 \pm 1.23$ \\
\hline Experiment 3: skill & (As above) & (As above) & $22.04 \pm 0.43$ & $23.48 \pm 0.49$ & (As above) \\
\hline
\end{tabular}

Table 2. Shown are the mean \pm SEM of MEP amplitudes $(\mathrm{mV})$ of the unconditioned test pulse intensity measured across all CBI time points

$\mathrm{CBI}$ unconditioned test pulse MEP amplitude (mV)

\begin{tabular}{clll}
\hline $\begin{array}{c}\text { Experiment } 1 \\
\text { PA }\end{array}$ & $1.13 \pm 0.08$ & & \\
AP & $1.05 \pm 0.11$ & & \\
Experiment 2 & Pre-PAS & Post-PAS & \\
PA & $1.19 \pm 0.10$ & $1.12 \pm 0.08$ & \\
AP & $1.10 \pm 0.09$ & $1.15 \pm 0.11$ & \\
Experiment 3 & & & P2 \\
Sequence & Baseline & P1 & $1.02 \pm 0.07$ \\
PA & $1.08 \pm 0.07$ & $1.09 \pm 0.09$ & $1.06 \pm 0.08$ \\
AP & $0.98 \pm 0.09$ & $1.01 \pm 0.08$ & $0.98 \pm 0.06$ \\
Skill & & & $0.92 \pm 0.09$ \\
PA & $1.03 \pm 0.08$ & $1.07 \pm 0.07$ & \\
AP & $0.94 \pm 0.08$ & $0.96 \pm 0.09$ & \\
\hline
\end{tabular}

We adjusted the stimulator intensity to attempt to keep this pulse consistent (i.e., $\sim 1 \mathrm{mV}$ ) throughout each experiment. We did not observe any changes across stimulation time points or between coil orientations that could be attributed to plastic changes either via PAS for $21.5 \mathrm{~ms}$ (experiment 2) or motor learning (experiment 3).

double-cone coil over the marked cerebellar target (the location was marked on the participant's head).

To measure CBI, the averaged MEP amplitudes of 15 single-pulse TMS responses over M1 were compared with the 15 paired-test plus conditioned responses. This was done for each CBI assessment across all experiments. When changes in M1 excitability occurred via either a plasticity protocol (experiment 2) or a motor learning task (experiment 3), the intensity of stimulation for the TS was adjusted to elicit similar MEP amplitudes (Table 2). Trials with any preceding background muscle activity was excluded. Moreover, any MEPs below the $50 \mu \mathrm{V}$ threshold were excluded from the analysis.

Paired associative stimulation. We used paired associative stimulation (PAS) as a protocol to induce plastic changes in M1 (Stefan et al., 2000; Wolters et al., 2003). PAS consisted of 200 electrical stimuli of the right ulnar nerve paired with consecutive TMS pulses over the FDI hot spot at a rate of $0.2 \mathrm{~Hz}$. Electrical stimulation was applied through a bipolar electrode using a constant current square wave pulse (duration, $1 \mathrm{~ms}$ ) at an intensity of $3 \times$ the perceptual threshold (Digitimer). TMS pulses were delivered with PA currents and with an intensity of $120 \%$ of rMT. We opted to test PAS pairings with the electrical stimuli preceding the PA-M1 TMS by $21.5 \mathrm{~ms}$, as this interval has been suggested to not depend on the cerebellum (Hamada et al., 2012). Indeed, the aftereffects of PAS applied at longer intervals (i.e., $25 \mathrm{~ms}$ ) can be blocked with cerebellar transcranial direct current stimulation, whereas the effects of a PAS of $21.5 \mathrm{~ms}$ remained unchanged. Thus, it is speculated that the afferent pathway from the stimulated nerve to M1 traverses the cerebellum in PAS $25 \mathrm{~ms}$, whereas a PAS of $21.5 \mathrm{~ms}$ is thought to represent direct interactions of lemniscal inputs.

Behavioral tasks

Sequential visuomotor isometric pinch-force task. We used the Sequential Visual Isometric Pinch Task (SVIPT) to assess motor skill learning (Reis et al., 2009; Marquez et al., 2013; Cantarero et al., 2015; Spampinato and Celnik, 2017, 2018). Participants were seated in front of a vertical 20 inch computer screen monitor and held a force transducer between the thumb and the index finger of the right hand. Isometrically squeezing the force transducer controlled the rightward movement of an on-screen cursor. The objective of the task was to move the cursor between a HOME position and five targets (HOME-1-HOME-2-HOME-3HOME-4-HOME-5). To move the cursor effectively, participants had to learn the logarithmic relationship between pinch force production and cursor movement. We quantified the amount of motor skill learning (SVIPT) by assessing changes in the speed-accuracy trade-off function (SAF). To do this, we used the following equation to estimate SAF throughout performance (i.e., skill measure):

$$
\text { Skill Measure }=\frac{1-\text { error rate }}{\text { error rate }\left(\ln (\text { movement time })^{b}\right)},
$$

where the error rate was defined as the proportion of unsuccessful trials (defined as the number of trials with at least one undershooting or overshooting movements), movement time was calculated as the total trial time (time between movement onset and when the cursor reached the final target), and parameter $b$ was fixed at 5.424, a value determined from an independent sample of subjects who performed the same task (for more details, refer to the supplementary text of Reis et al., 2009). Error rate and movement time parameters were averaged for each block consisting of 30 consecutive trials.

Sequence training. Participants performed on a nine-element sequence task requiring responses to visually cued boxes on a computer screen (Spampinato and Celnik, 2018). They were instructed to generate responses to the sequentially ordered visual cues by pressing their index finger on a directly mapped computer key. Sequence targets were displayed using a horizontal display of three square stimuli, representing a direct left-to-right mapping of three neighboring keys ("Z," leftmost; " $X$," middle; " $C$," rightmost). The sequence CZXZCXCXZ was presented: and was kept identical for all subjects. A trial began with a fixation cross, which was displayed for $2 \mathrm{~s}$. Participants were then instructed to respond to the cued stimulus as quickly as possible. Importantly, only when the correct response was selected would the next target in the sequence become highlighted. In other words, if an incorrect response was made, the sequence was paused and only resumed following the appropriate key response. After 10 consecutive trials, participants were given feedback on their performance (average movement time and error-rate) and were informed to improve their score on the subsequent trials.

\section{Experimental procedures}

Experiment 1 . We tested how applying cerebellar stimulation at varying ISIs to distinct M1 coil orientations (i.e., probing PA vs AP currents) affected CBI. Thus, we tested both AP-CBI and PA-CBI at ISIs of 3, 5, and $7 \mathrm{~ms}$. Specifically, we selected these intervals as the suppressive effects measured between ISIs of 5 and $7 \mathrm{~ms}$ have suggested to be cerebellar (Werhahn et al., 1996; Fisher et al., 2009; Fig. 1A). Intervals $>7 \mathrm{~ms}$ are likely to result in the suppression from peripheral nerve fibers in the brachial plexus (Werhahn et al., 1996; Hardwick et al., 2014), whereas intervals $<5 \mathrm{~ms}$ do not produce strong suppressive effects likely due to the amount of time needed for cerebellar stimulation to hit cortical targets. Thus, $3 \mathrm{~ms}$ was used as a control. For each coil orientation, we recorded 15 responses for all four conditions, as follows: one corresponding to the TS over M1 alone and for CBI measured the three different ISIs. The M1 test pulse intensity was adjusted to elicit a $\sim 1 \mathrm{mV}$ MEP 
A

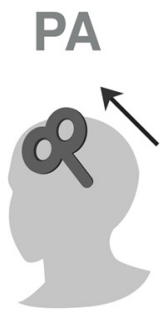

AP

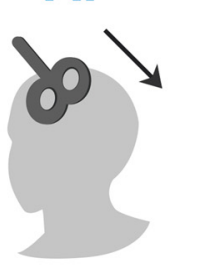

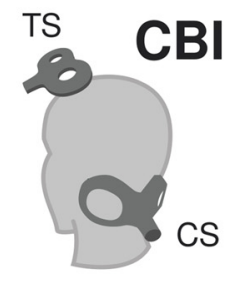

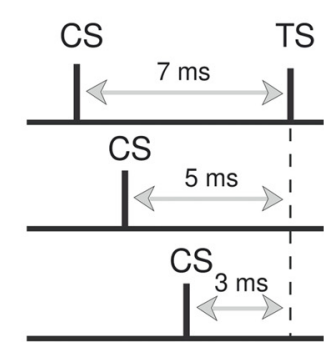

B

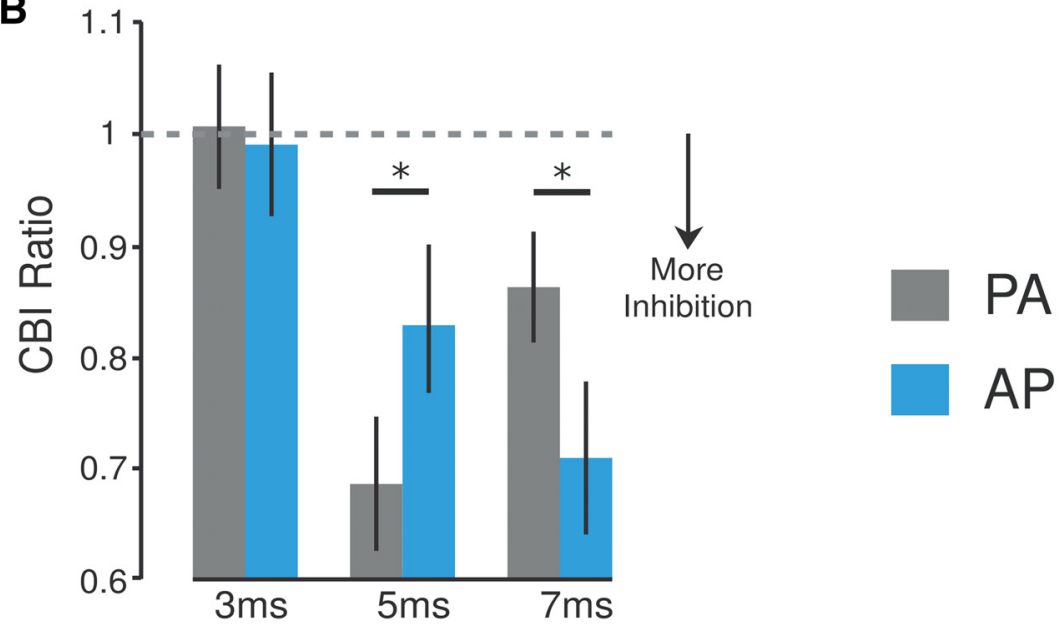

Figure 1. Effects of different current directions applied over M1 on cerebellar-M1 connectivity (CBI). A, Schematic representation of experiment 1. This experiment tested how applying cerebellar stimulation (i.e., $(S)$ at varying interstimulus intervals before applying a TS to distinct M1 coil orientations (PA vs AP currents) affected CBI. Thus, we tested both PA-CBI and AP-CBI at ISIs of 3, 5, and $7 \mathrm{~ms}$. $\boldsymbol{B}$, Bar graphs and vertical error bars depict the mean \pm SEM $\left({ }^{*} p \leq 0.05\right)$ of the $(B \mid$ ratio. The $x$-axis represents different ISls $(3,5$, and $7 \mathrm{~ms})$ applied for both $\mathrm{PA}-\mathrm{CBI}$ and $\mathrm{AP}$-CBI. The $y$-axis shows the $\mathrm{CBI}$ as the ratio of the conditioned versus the unconditioned MEP. Ratio values $<1$ represent inhibition, whereas ratios $>1$ represent facilitation. CBI values measured with the different M1 currents were matched for test MEP amplitude values $\sim 1 \mathrm{mV}$. Here, we found that PA-CBI measured with an $I S \mathrm{I}$ of $5 \mathrm{~ms}$ elicited stronger CBI than AP-CBI. On the other hand, when measured at $7 \mathrm{~ms}$, AP produced a significantly larger effect than PA-CBI. Importantly, we did not find any evidence of CBI for either M1 current direction when measured at a $3 \mathrm{~ms} I \mathrm{SI}$ since this interval is presumably too short to elicit any cerebellar effects to cortical regions.

amplitude unconditioned response for each coil orientation. The magnitude of CBI was computed as the ratio of the conditioned MEP over the unconditioned MEP. A similar experimental procedure was conducted in a control study. Here, the M1 intensities for each current direction were matched (Fig. 2).

Experiment 2. To determine whether the two separate cortico-neuronal networks process cerebellar inputs in distinct ways, we administer a plasticity protocol to M1 PA inputs. To do this, we measured AP-CBI and PA-CBI values at their preferential ISI, before and after standard PAS plasticity protocol (Fig. $3 A$ ). Importantly, we administered the repeated pairs of electrical stimuli to the ulnar nerve and PA-TMS at an interval of $21.5 \mathrm{~ms}$ (i.e., PAS at $21.5 \mathrm{~ms}$ ) since this technique can modulate the plasticity of PA-M1 excitability without affecting cerebellar activity (Hamada et al., 2014). Fifteen responses were recorded for the orientation-related ISI condition (e.g., $5 \mathrm{~ms}$ for PA, $7 \mathrm{~ms}$ for AP) and 15 responses for the TS alone.

Experiment 3. Subjects participated in a counterbalanced crossover design in which participants trained on the SVIPT in one session and on the sequence training in another session (Fig. $4 A$ ). The order of training sessions was randomized and separated by at least $48 \mathrm{~h}$. For each motorlearning task, participants completed 150 trials (five blocks; one block $=30$ trials) where movement times and accuracy measures were recorded. In addition to behavioral measurements, we investigated how learning two distinct motor tasks affected AP-CBI and PA-CBI at their preferential ISIs ( 5 and $7 \mathrm{~ms}$, respectively).To do this, we measured CBI (assessed with the different M1 current inputs) before, during, and after individuals learned each task.

\section{Data analysis}

For all data statistical analyses, SPSS (version 20; IBM) was used and effects were considered significant at $p \leq 0.05$. All data are given as the mean \pm SEM. We used separate polynomial nested repeated-measures ANOVA ( ANOVA $_{\mathrm{RM}}$ ) for all behavioral and physiological measures. When significant differences were identified, we used BonferroniHolm-corrected post hoc analysis to account for multiple comparisons.

Experiments 1 and 2. To determine changes in $\mathrm{CBI}$ in experiment 1 , we used ANOVA $_{R M}$ with within-subject factors ORIENTATION (PA, AP) and ISI $(3,5,7 \mathrm{~ms})$. To determine whether PAS modulates baseline M1 excitability and $\mathrm{CBI}$ responses between the two coil orientations, we performed ANOVA $A_{\mathrm{RM}}$ with factors TIME (PRE, POST) and ORIENTATION (PA, AP).

Experiment 3. To assess the performance of the SVIPT, we measured differences in the skill measure by using ANOVA $_{\text {RM }}$ with TIME (Block1, Block2 ... Block5) as a within-factors measure. To assess sequence learning, we compared differences in the online error rate and movement time. We used two separate $\mathrm{ANOVA}_{\mathrm{RM}}$ for these measures with TIME (Block1, Block2 ... Block5) as the within-subject factor. To determine changes in CBI between the two coil orientations, we used separate ANOVA $_{\mathrm{RM}}$ with between-subjects factor GROUP (Training, Random) and within-subject factors TIME (PRE, P1, P2) and ORIENTATION (PA, AP). Here, GROUP represents whether individuals were given a learnable task (Training) compared with ones given a randomized version of the task (Random; i.e., no motor learning is expected). TIME represents distinct stimulation time points before training (PRE), after one block of training (P1), and at the end of five training blocks (P2).This was done separately for each task.

\section{Results}

Experiment 1 results: PA-CBI measured at $5 \mathrm{~ms}$ ISI and AP$\mathrm{CBI}$ at $7 \mathrm{~ms}$ ISI elicits the largest $\mathrm{CBI}$ response

We investigated the effect of M1 coil orientation (PA and AP currents) on cerebellar-M1 connectivity responses by measuring

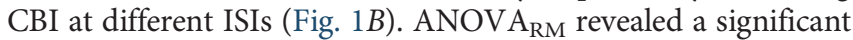
main effect of ISI $\left(F_{(2,44)}=17.947, p=0.001\right)$ and ISI $\times$ ORIENTATION interaction $\left(F_{(2,44)}=6.556, p=0.003\right)$. Post hoc analysis revealed CBI responses measured with PA currents at an ISI of 5 and $7 \mathrm{~ms}$ were significantly different when compared with measures of CBI at an ISI of $3 \mathrm{~ms}$ (respectively, $p=0.001$ and $p=0.017)$. On the other hand, for AP current inputs to M1, CBI was only observed at an ISI of 7 ms when compared with an ISI of $3 \mathrm{~ms}(p=0.001)$, while no difference was found between 5 and $3 \mathrm{~ms}(p=0.117)$. This indicates that M1 AP currents are more responsive to cerebellar stimulation when a longer interval is given between stimulation sites. Interestingly, the post hoc analysis also revealed differences between the coil orientations at different ISIs. Here, we found that CBI measured at $5 \mathrm{~ms}$ was 


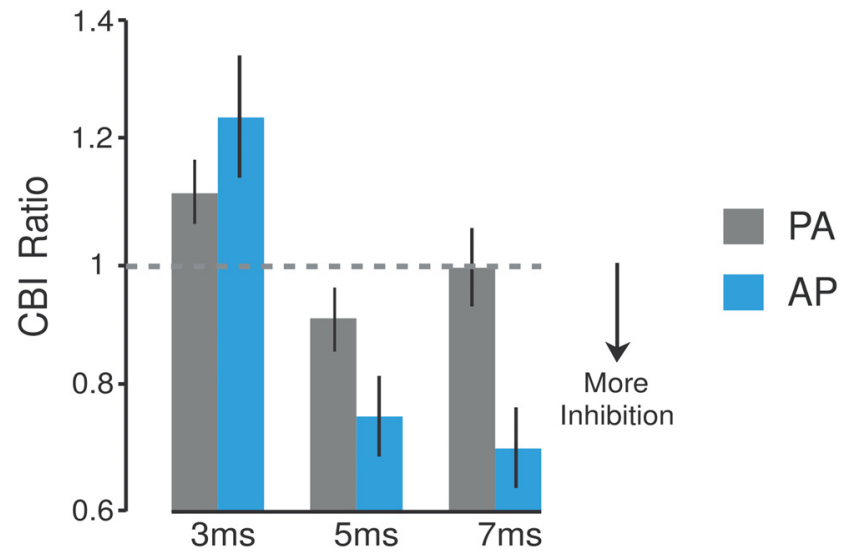

Figure 2. Matched current intensity control experiment. Schematic representation of the control experiment $(n=6)$. This study tested whether the $C B I$ differences found in experiment 1 can be explained by the different $P A$ and $A P$ stimulation intensities required to elicit a matched $\sim 1 \mathrm{mV}$ test MEP response (Table 1 ). In this experiment, $\mathrm{PA}-\mathrm{CB}$ and $\mathrm{AP}-\mathrm{CB}$ I were retested at ISIs of 3, 5, and 7 ms using the same stimulation intensity for each current direction. The selected intensity was based on the lowest test MEP amplitude value possible to elicit a CBI effect ( $\sim 0.5 \mathrm{mV}$ for the AP current; for review, see Pinto and Chen, 2001). Bar graphs and vertical error bars depict the mean \pm SEM of the CBI ratio. The $x$-axis represents different ISIs (3, 5, and $7 \mathrm{~ms}$ ) applied for both PA-CBI and AP-CBI. The $y$-axis shows CBI as the ratio of the conditioned versus the unconditioned MEP. Ratio values $<1$ represent inhibition, whereas ratios $>1$ represent facilitation. The mean \pm SEM PA and AP test MEP amplitude values recorded for this experiment were $2.56 \pm 0.42$ and $0.60 \pm 0.15 \mathrm{mV}$, respectively.

more prominent for PA currents $(p=0.039)$, whereas AP currents elicited a stronger effect at $7 \mathrm{~ms}(p=0.025)$. Importantly, we found no evidence of CBI for either M1 current direction when measured at an ISI of $3 \mathrm{~ms}(p=0.361)$. The results from experiment 1 demonstrate that assessing cerebellar-M1 connectivity at different timings between TMS pulses and with different coil orientations over M1 elicits distinct CBI responses.

It is important to consider that these results may be explained by the different TMS intensities required for the two current directions to elicit a matched $\sim 1 \mathrm{mV}$ test MEP response (Table 1). Indeed, these differences could potentially be responsible for activating different cortical circuits. As such, we conducted a control experiment in which the same procedure of experiment 1 was conducted but with matching the PA and AP stimulation intensities (Fig. 2). To do this, we recruited six individuals in which the PA-TMS and AP-TMS thresholds were relatively similar (i.e., $>10 \%$ stimulator output difference) as too large of a test MEP amplitude leads to a diminished CBI response (Pinto and Chen, 2001). To determine the changes in CBI for this control experiment, we used $\mathrm{ANOVA}_{\mathrm{RM}}$ with within-subject factors ORIENTATION (PA, AP) and ISI (3, 5, $7 \mathrm{~ms})$. Here, the ANOVA $_{\mathrm{RM}}$ revealed a significant ORIENTATION $\times$ ISI interaction $\left(F_{(2,10)}=4.796, p=0.035\right)$. Specifically, post hoc analysis revealed that AP currents elicited a stronger $\mathrm{CBI}$ effect at $7 \mathrm{~ms}$ than PA currents $(p=0.007)$, whereas the same difference was not found for $3 \mathrm{~ms}(p=0.321)$ or $5 \mathrm{~ms}(p=0.148)$. We also found that within the CBI responses measured with PA currents, PACBI values measured at $5 \mathrm{~ms}$ were significantly different when compared with those measured at $3 \mathrm{~ms}(p=0.001)$. Together, these results mimic the findings of experiment 1 , illustrating that the most prominent $\mathrm{CBI}$ for PA currents is found at $5 \mathrm{~ms}$, whereas the strongest $\mathrm{CBI}$ for AP currents is at $7 \mathrm{~ms}$. The results of this control experiment demonstrate that the results of experiment 1 are not explained by the different TMS intensities required for each current direction.

\section{Experiment 2 results: PA-PAS modulates only PA-CBI}

In the next experiment, we sought to determine whether the results of experiment 1 reflects a distinct processing of cerebellar inputs within M1. To do this, we measured PA-CBI versus AP$\mathrm{CBI}$ at their preferential ISI, before and after standard PA-PAS. We asked whether applying standard PAS for $21.5 \mathrm{~ms}$ would result in orientation-specific changes in cerebellar-M1 connectivity (Fig. 3). Importantly, we selected PAS for $21.5 \mathrm{~ms}$ rather than PAS for $25 \mathrm{~ms}$, as this method is shown to depend on PA current directions, but not AP current directions (Hamada et al., 2014), and moreover, does not alter general cerebellar excitability that would likely modulate both PA-CBI and AP-CBI.

We first assessed whether PAS for $21.5 \mathrm{~ms}$ specifically modulated M1 excitability assessed with PA current directions. ANOVA $_{\mathrm{RM}}$ revealed a significant main effect of TIME $\left(F_{(1,14)}=\right.$ $4.838, p=0.047)$ and TIME $\times$ ORIENTATION interaction $\left(F_{(1,14)}=5.121, p=0.041\right)$. Post hoc analysis revealed that PAS for $21.5 \mathrm{~ms}$ increased M1 excitability when measured with PA currents $(p=0.008)$, whereas M1 excitability measured with AP currents remained unchanged $(p=0.493)$. We then compared PA-CBI and AP-CBI before and immediately after PAS for 21.5 ms (Fig. 3C). ANOVA ${ }_{\mathrm{RM}}$ revealed a significant main effect of $\operatorname{TIME}\left(F_{(1,14)}=4.484, p=0.043\right)$ and TIME $\times$ ORIENTATION interaction $\left(F_{(1,14)}=6.556, p=0.029\right)$. We found that PAS for $21.5 \mathrm{~ms}$ decreased PA-CBI $(p=0.04)$, but did not modulate AP$\mathrm{CBI}(p=0.47)$, indicating that $\mathrm{CB}-\mathrm{M} 1$ interactions are different for the two M1 neural networks. Importantly, this change was not due to the PAS-induced M1 excitability changes as we adjusted the stimulator intensity to evoke an MEP with a peakto-peak amplitude of $\sim 1 \mathrm{mV}(\mathrm{S} 1 \mathrm{mV})$ when recoding $\mathrm{CBI}$ for each current direction and time point (Fig. 3C, inset). ANOVA $_{\mathrm{RM}}$ confirmed that our adjustment of the test pulse intensity was valid across CBI time points as differences in test pulse amplitudes were not significantly different for factors ORIENTATION $\left(F_{(1,14)}=6.556, p=0.639\right)$ or $\operatorname{TIME}\left(F_{(1,14)}=\right.$ $6.556, p=0.563)$.

\section{Experiment 3 results}

Participants learned the skill and sequence tasks

To assess SVIPT skill learning, we quantified the skill score (Eq. 1 ), which incorporates the movement time and error rate for

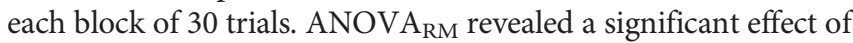
$\operatorname{BLOCK}\left(F_{(4,44)}=7.173 ; p<0.001\right)$. Specifically, post hoc analysis showed that individuals were better at performing the last block of training compared with the initial block $(p<0.045)$, indicating that participants improved skill performance within the training session (Fig. 4B). Additionally, to assess learning in the sequence task, we calculated the average movement time and error rate for each training block of 30 sequence trials (Fig. 4C). ANOVA $_{\mathrm{RM}}$ revealed that participants were able to improve their movement time $\left(F_{(4,44)}=61.859 ; p<0.001\right)$ without compromising changes in their performance error rate $\left(F_{(4,44)}=0.619\right.$; $p=0.651)$.

\section{$P A-C B I$ is decreased early in skill learning, whereas AP-CBI is} reduced only late in skill learning

We compared the amount of cerebellar-M1 connectivity changes for each orientation (PA-CBI, AP-CBI) before skill learning (Pre), following one block of training (P1) and after five blocks of training (P2; Fig. $4 D)$. ANOVA RM $_{\text {revealed a significant }}$ CBI changes for TIME $\left(F_{(2,44)}=5.807 ; p=0.006\right)$ and TIME $\times$ GROUP interaction $\left(F_{(2,44)}=7.801 ; p=0.001\right)$. PA-CBI showed a selective reduction following early skill learning when compared 
A
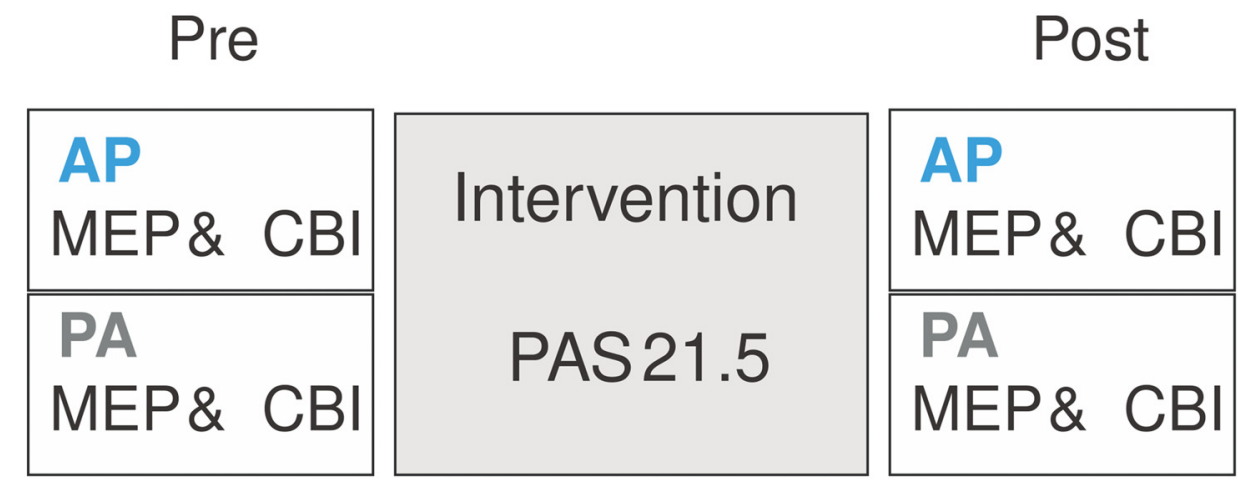

B

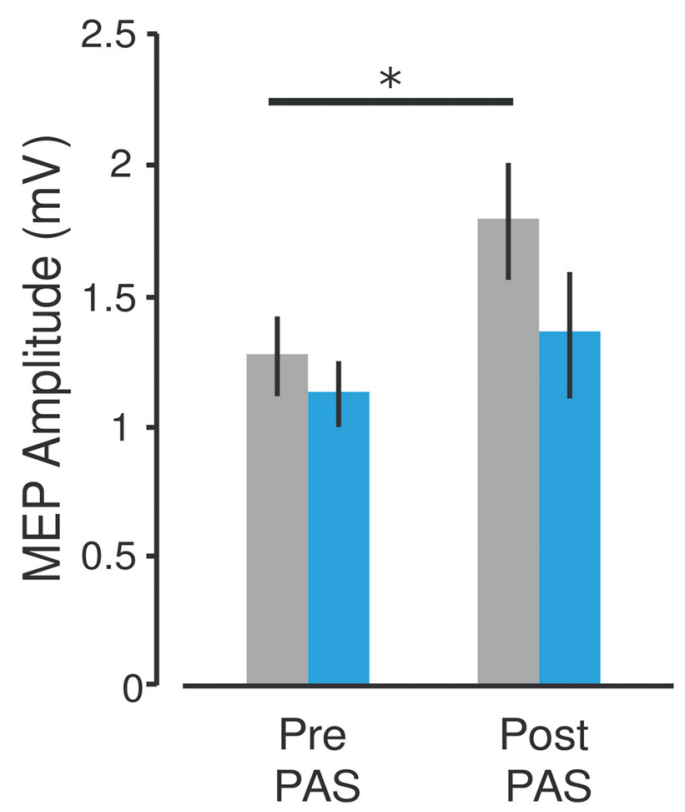

C
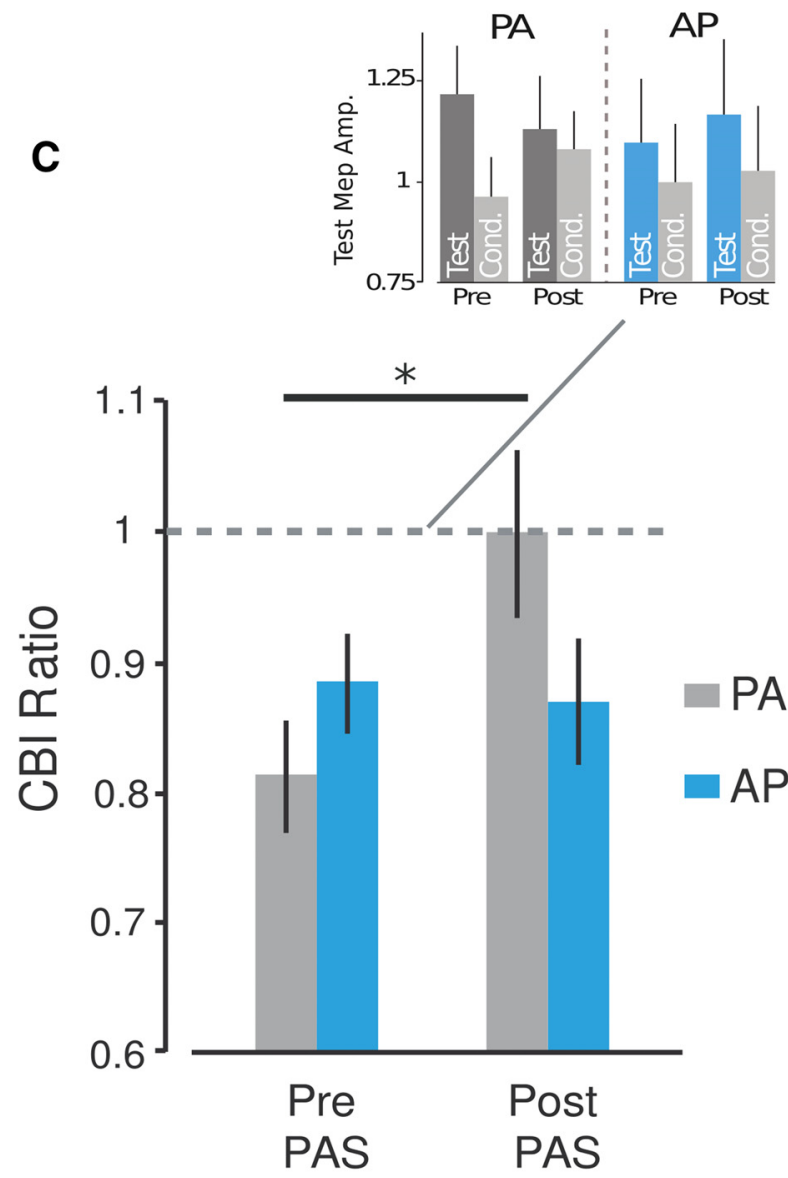

Figure 3. PAS only modulates PA-CBI, and not AP-CBI. A, Schematic representation of experiment 2. For each current direction, $M 1$ excitability and CBI was measured before and after participants received PAS. $\boldsymbol{B}, \boldsymbol{C}$, PAS effects on M1 excitability $(\boldsymbol{B})$ and $\mathrm{CBI}(\boldsymbol{C})$. Bar graphs and vertical error bars depict the mean $\pm S E M,{ }^{*} p \leq 0.05$, where data presented with gray colors represent PA currents applied to M1 and blue colors represent AP currents. The $y$-axis shows the MEP amplitudes $(\boldsymbol{A})$ and $C B$ ratio values $(\boldsymbol{B})$, while the $x$-axis displays these values before (pre) and after (post) administration of PAS protocol. $\boldsymbol{B}$, We found that only MEP amplitudes assessed with PA currents significantly increased with PAS. C, Similarly, we show that PA-CBI, and not AP-CBI, is decreased due to the PAS protocol. Importantly, this change was not due to the PAS-induced M1 excitability changes (i.e., results above) as we adjusted the S1mV when recording CBI for each current direction and time point (inset).

with baseline $(p=0.006)$, and in addition was significantly different compared with the value of AP-CBI early on in learning the task $(p=0.037)$. On the other hand, AP-CBI specifically reduced at the end of skill training when compared with both baseline $(p=0.013)$ and P1 $(p=0.024)$, and furthermore was found to be significantly different from the PA-CBI changes found at the end of training ( $p=0.047$ ). This result suggests that the reduction of CBI assessed for each coil orientation is sensitive to the amount of training individuals undergo when acquiring a new skill.
$P A-C B I$ is only reduced in early motor sequence learning

As previous investigations showed that $\mathrm{CBI}$ changes in more simplistic motor sequence learning mimic the changes found in more complex motor skill learning (Spampinato and Celnik, 2018), we also test whether AP-CBI and PA-CBI changed differently when learning a simple sequence. We observed a significant TIME $\times$ GROUP interaction of these factors $\left(F_{(2,26)}=3.49\right.$, $p<0.05)$. Here, we found that only PA-CBI was significantly reduced following early sequence training (P1) when compared 
with baseline responses $(p=$ 0.013). This effect was also significantly different from AP-CBI changes following early sequence learning $(p=0.036)$. These results indicate that only cerebellar-M1 connectivity measured with PA over M1 is sensitive to learning motor sequences (Fig. 4D).

\section{Discussion}

The present study directly assessed cerebellar-M1 connectivity using CBI. We found that output from the cerebellum interacts differentially, with the M1 networks activated preferentially by $\mathrm{PA}$ and $\mathrm{AP}$ current pulses. This is, in part, consistent with prior investigations that used cerebellar TDCS as an indirect method to probe cerebellar-M1 interactions (Hamada et al., 2014). Specifically, we found that CBI produced maximum suppression of MEPs evoked by PA pulses at an interstimulus interval of $5 \mathrm{~ms}$, whereas it was maximum at $7 \mathrm{~ms}$ for AP-MEPs. Moreover, paired associative stimulation of ulnar nerve input with PA-MEPs suppressed the CBI of PA-MEPs but did not change AP-MEPs. Finally, $\mathrm{CBI}$ to the AP-sensitive and PAsensitive networks was modulated in unique ways depending on the type of motor skill task individuals were required to learn. We argue below that this is consistent with the idea that different cortical motor circuits are involved in different behaviors and that they interact with input from the cerebellum in distinct ways.

\section{CBI of AP-evoked and PA-evoked MEPs: two different pathways?}

The cerebellum is a critical part of the motor network involved in learning new skills that integrate both motor and cognitive components. This notion is supported by the known mass connections the cerebellum has to both motor and nonmotor regions of the cerebral cortex through cerebello-thalamo-cortical pathways (Kelly and Strick, 2003; Bostan et al., 2013). More-over, evidence from electrophysiological recordings has shown that these pathways are quite complex: the axons of these pathways can terminate on both excitatory and inhibitory neurons (Na et al., 1997; Daskalakis et al., 2004), via disynaptic or polysynaptic connections (Yamamoto et al., 1984; Futami et al., 1986; Holdefer et al., 2000), and innervate across cortical layers I, III, V,
A

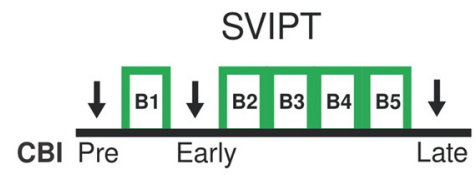

B

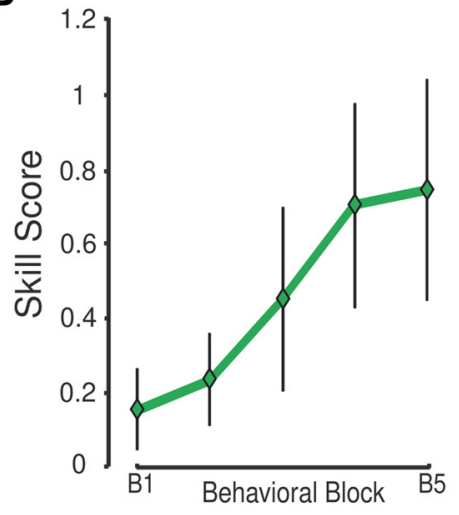

C

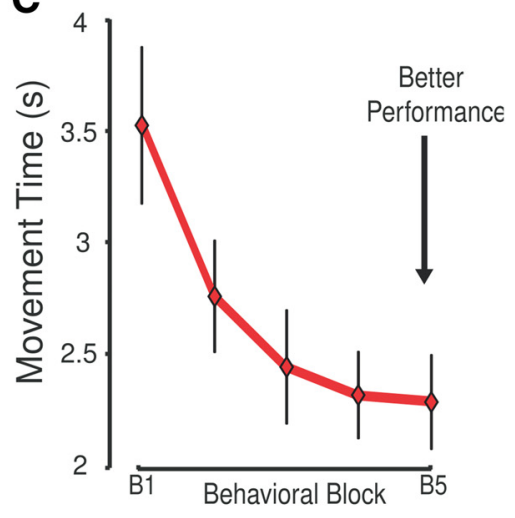

D
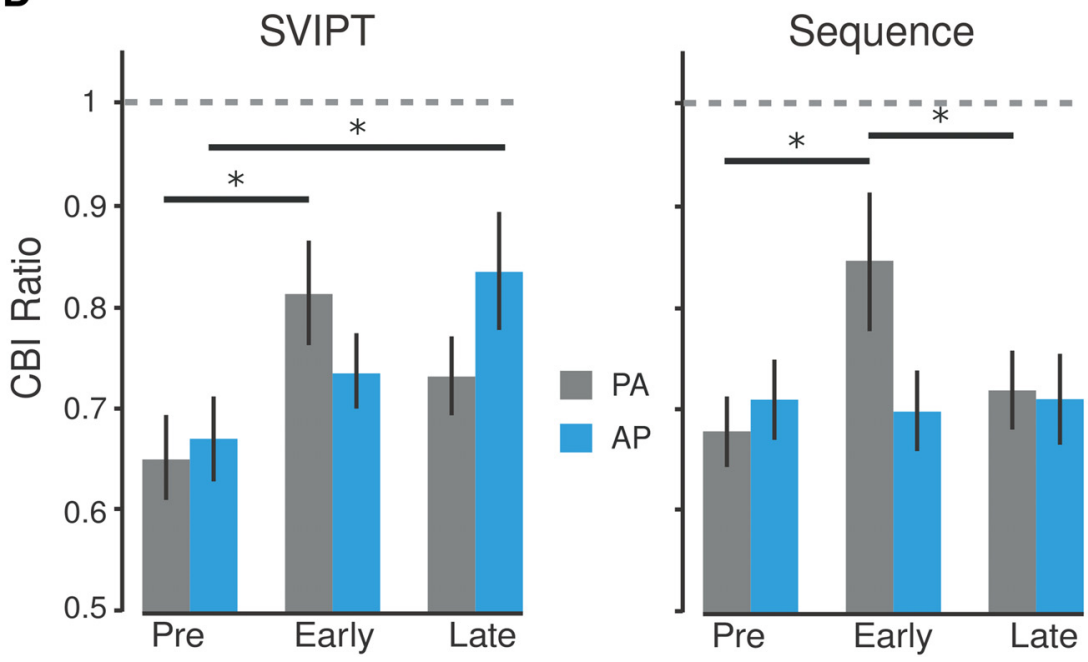

Figure 4. $\quad \mathrm{PA}-\mathrm{CBI}$ and $\mathrm{AP}-\mathrm{CBI}$ changes with learning distinct motor tasks. A, Schematic representation of the experiment. Participants completed five blocks consisting of 30 trials of a motor skill task (green) and of a simple sequential task (red). Cerebellar connectivity to both AP-sensitive and PA-sensitive interneurons was assessed before training (Pre) and after the first (P1) and final training behavioral block (P2). B, C, Behavioral results. B, Skill task results. The $x$-axis depicts training blocks (average of 30 trials), and the $y$-axis represents the average skill measure scores. Higher values depict a participant's ability to improve their speed-accuracy trade-off performance of the task. The data represent the mean \pm SEM for each block. C, Sequence learning results. The $x$-axis depicts training blocks (average of 30 trials) and the $y$-axis represents the average movement time to perform the entire sequence. Of note, participants were able to improve their movement time while maintaining the same level of accuracy. $\boldsymbol{D}$, Cerebellar-M1 connectivity changes. Bar graphs and vertical error bars depict the mean \pm SEM $\left({ }^{*} p \leq 0.05\right)$ of the (BI ratio when measured with AP-M1 (blue) and PA-M1 (gray) currents at each stimulation time-points (Pre, P1, P2). Dashed horizontal line depicts the normalized unconditioned MEP amplitude, and the dashed vertical line represents the separation between different motor learning tasks. Of note, $C B I$ for each $M 1$ current direction was measured at their preferred ISI (i.e., $7 \mathrm{~ms}$ for AP-CBI; $5 \mathrm{~ms}$ for PA-CBI). We found decreases in PA-CBI that occurred early on for both the motor skill and sequence tasks; however, AP-CBI was reduced only later as individuals optimized their motor skill performance.

and VI (Ando et al., 1995; Na et al., 1997). Anatomically, cerebellar projections to leg and arm areas of M1 arise in different parts of the dentate nucleus, as do projections to premotor and other frontal regions (Dum and Strick, 2003).

Cerebellar TMS is unlikely to be very selective and probably engages all of these intricate pathways, perhaps via the recruitment of Purkinje cells. Since these have a suppressive influence on deep cerebellar nuclei (Celnik, 2015), cerebellar TMS will tend to reduce any ongoing activity in these connections to M1. Our results show that the time at which this has maximum effect 
on M1 is different for the networks activated by PA and AP current pulses and is compatible with the idea that there are separate connections from the cerebellum onto these networks with different conduction times. It should be noted that the latency of MEPs evoked by AP stimulation is $2-3 \mathrm{~ms}$ longer than for $\mathrm{P}$ -stimulation, which is similar to the difference in optimal CBI timing. However, if the time of maximum CBI had been related to MEP latency, then we would have expected the best interval for CBI of AP-MEPs would have been 2-3 ms earlier than the $\mathrm{CBI}$ of PA-MEP rather than 2-3 ms later.

A recent modeling study suggests that whereas PA pulses preferentially activate the synaptic terminals of layer 5 pyramidal neurons located in the rostral lip of the central sulcus, AP pulses activate terminals in the crown of the gyrus that could originate in premotor regions (Aberra et al., 2020). If so, then it is possible that PA-CBI directly targets the excitability of M1 layer 5 pyramidal neurons and reduces the amplitude of PA-evoked MEPs. In contrast, AP-CBI could target the excitability of neurons in the premotor cortex that project to M1. Reducing the tonic level of excitation in these pathways could then reduce the excitability of the gyral M1 neurons that receive input from the synaptic terminals of premotor axons activated by AP pulses. The additional time for cerebellar inputs to traverse the premotor cortex would mean that AP-MEPs would be maximally suppressed at longer ISIs than PA-MEPs. Retrovirus tacking studies in nonhuman primates have revealed cerebellar projections to prefrontal, dorsal, and ventral premotor, supplementary motor, and parietal areas (Kelly and Strick, 2003), and in macaques inputs have been demonstrated from Purkinje cells to area F2r, an area considered similar to dorsal premotor cortex in humans (Hashimoto et al., 2010). Moreover, projections of the cerebellar dentate to the arm areas of M1 and premotor cortex are anatomically divided as distinct output channels (Middleton and Strick, 2000; Bostan et al., 2013). Therefore, it is conceivable that at least two distinct connections (i.e., premotor and M1) from the cerebellum can be realized by changing the current direction applied with TMS.

Although the time for optimal CBI differed, the level of suppression of AP-MEPs and PA-MEPs was very similar. While this seems contrary to results of Hamada et al. (2012, 2014), who found that TDCS over cerebellum suppressed AP-MEPs more than PA-MEPs, suggesting that CBI is stronger to AP networks. However, it is difficult to compare the two results. TDCS can affect M1 excitability through the entire complex network of cerebellar inputs to M1. Its effect on MEPs is the sum of all these effects. In contrast, $\mathrm{CBI}$ only gives information on the most rapid connections, making it impossible to compare the two.

\section{Evidence from paired associative stimulation}

In the second experiment, we examined the effect of PAS for $21.5 \mathrm{~ms}$ on CBI. The facilitatory aftereffects of PAS for $21.5 \mathrm{~ms}$ are thought to depend on PA networks rather than AP networks (Hamada et al., 2012, 2014). As expected, we found a modulation of PA-CBI with no effect on the amount of CBI tested with APMEPs, confirming the selectivity of PAS for 21.5 md to PA networks. However, we found that this intervention suppressed the PA-CBI effect. We can only speculate on the mechanism involved in this. It may relate to the fact that the PAS method repeatedly pairs a single volley of afferent input that is carefully timed to arrive just before the TMS pulse. However, other inputs (e.g., those from the cerebellum) occur randomly and have no specific timing relationship to TMS or afferent input. It could be that under these circumstances, the strength of these inputs is reduced by PAS with the effect that CBI is no longer effective.
Whatever the mechanism, the results again suggest that there are two distinct cerebellar-cortical neuronal networks.

\section{Evidence from motor learning}

Previous investigations have suggested that separate interneuron circuits within M1 have distinct roles in how they contribute to learning a variety of motor behaviors (Hamada et al., 2014). Specifically, cerebellar-dependent error-based forms of learning engage interneurons activated by AP-M1 TMS. Here, we had individuals learn two distinct motor tasks that likely involve the cerebellum in different ways and tested whether it changed the $\mathrm{CBI}$ connectivity to AP versus PA networks. One task required individuals to learn only a sequence of movements, whereas the other task required individuals to learn how to control a new device in a novel environment (i.e., sensorimotor map), along with performing a sequence of movements. The latter skill has been suggested to rely more heavily on cerebellar-dependent errorbased learning to learn the dynamics of the skill task (Diedrichsen et al., 2010; Taylor and Ivry, 2014). Indeed, although learning a sequence of movements can elicit changes in cerebellar excitability, these changes were previously only found to modulate activity at the initial stage of learning (Spampinato and Celnik, 2018). The results of the present study are consistent with those of prior studies as the PA-CBI effect changes early on for both skill tasks, whereas AP-CBI is reduced only after individuals begin to optimize their performance of the complex skill, suggesting that the motor-learning processes engaged in this task differentially recruit the pathways probed with directional TMS.

A possible explanation for the present results is that PA and AP inputs to cortical-spinal neurons have different roles in learning the different tasks. For instance, the early changes in PA-CBI occur during a period in which performance is initially quite poor. This could be a time during which cerebellar involvement in both tasks could reflect the activity of an error correction mechanism (Shadmehr et al., 2010), supporting the idea that the cerebellum updates motor commands during error-dependent learning (Herzfeld et al., 2014). The later changes in AP-CBI perhaps indicate that performance is being optimized. The cerebellum has been implicated in "automatizing" behavior (Balsters et al., 2013) in tasks where extended practice lessens the overall cognitive demand to perform a task (i.e., performed entirely implicitly; Doyon and Benali, 2005). Interestingly, recent work in rodents has revealed that firing rates within the cerebellum and premotor cortex become strongly coupled only as behavioral performance improves, although they are initially dissimilar (Wagner et al., 2019). The results would therefore be consistent with our hypothesis that AP-CBI operates via cerebellar inputs to premotor cortex. As the premotor cortex has been previously found as a neural substrate flexible for recombination and efficient encoding of complex motor behaviors, it is possible that inputs to this region from the cerebellum may help to shape the overall representation of the task in a behavior where learning a new sensorimotor mapping is required.

\section{Implications and limitations}

These results do not come without some limitations. First, it remains unclear exactly what and where TMS stimulates. Thus, the idea that currents applied in the AP direction over M1 may recruit premotor inputs remains speculative. Future studies may consider investigating the effect of applying a "virtual" lesion to premotor areas to see whether pathways interacting with AP currents are specifically modulated. Second, we cannot disentangle whether the differences in AP-CBI and PA-CBI found here reflect two anatomically distinct sets of cerebello-cortical fibers 
or whether the differences found here are due to the same cerebellar input being processed differently by two distinct populations of cortical neurons. Finally, our explanation as to why these two distinct circuits behave differently when assessing interactions between PAS and motor learning is speculative and will require further study. Despite these limitations, we suggest that two distinct cerebellar-cerebral interactions can be disentangled using cerebellar stimulation in combination with directional TMS over the cerebral cortex. Our hypothesis is that these pathways can contribute to independent processes of learning, depending on the behavior individuals are required to learn. They also present a novel approach to probing connections between the cerebellum and cerebral cortex that can be used in future patient studies to understand the pathophysiology of diseases in which the cerebellum is implicated to play a functional role.

\section{References}

Aberra AS, Wang B, Grill WM, Peterchev AV (2020) Simulation of transcranial magnetic stimulation in head model with morphologically-realistic cortical neurons. Brain Stimul 13:175-189.

Ando N, Izawa Y, Shinoda Y (1995) Relative contributions of thalamic reticular nucleus neurons and intrinsic interneurons to inhibition of thalamic neurons projecting to the motor cortex. J Neurophysiol 73:2470-2485.

Argyelan M, Carbon M, Niethammer M, Ulug AM, Voss HU, Bressman SB, Dhawan V, Eidelberg D (2009) Cerebellothalamocortical connectivity regulates penetrance in dystonia. J Neurosci 29:9740-9747.

Balsters JH, Whelan CD, Robertson IH, Ramnani N (2013) Cerebellum and cognition: evidence for the encoding of higher order rules. Cereb Cortex 23:1433-1443.

Bostan AC, Dum RP, Strick PL (2013) Cerebellar networks with the cerebral cortex and basal ganglia. Trends Cogn Sci 17:241-254.

Cantarero G, Spampinato D, Reis J, Ajagbe L, Thompson T, Kulkarni K, Celnik P (2015) Cerebellar direct current stimulation enhances on-line motor skill acquisition through an effect on accuracy. J Neurosci 35:3285-3290.

Celnik P (2015) Understanding and modulating motor learning with cerebellar stimulation. Cerebellum 14:171-174.

Daskalakis ZJ, Paradiso GO, Christensen BK, Fitzgerald PB, Gunraj C, Chen R (2004) Exploring the connectivity between the cerebellum and motor cortex in humans. J Physiol 557:689-700.

Day BL, Dressler D, Maertens de Noordhout A, Marsden CD, Nakashima K, Rothwell JC, Thompson PD (1989) Electric and magnetic stimulation of human motor cortex: surface EMG and single motor unit responses. J Physiol 412:449-473.

Delvendahl I, Lindemann H, Jung NH, Pechmann A, Siebner HR, Mall V (2014) Influence of waveform and current direction on short-interval intracortical facilitation: a paired-pulse TMS study. Brain Stimul 7:49-58.

Di Lazzaro V, Rothwell JC (2014) Corticospinal activity evoked and modulated by non-invasive stimulation of the intact human motor cortex. J Physiol 592:4115-4128.

Di Lazzaro V, Ziemann U (2013) The contribution of transcranial magnetic stimulation in the functional evaluation of microcircuits in human motor cortex. Front Neural Circuits 7:18.

Di Lazzaro V, Restuccia D, Oliviero A, Profice P, Ferrara L, Insola A, Mazzone P, Tonali P, Rothwell JC (1998) Effects of voluntary contraction on descending volleys evoked by transcranial stimulation in conscious humans. J Physiol 508:625-633.

Di Lazzaro V, Oliviero A, Saturno E, Pilato F, Insola A, Mazzone P, Profice P, Tonali P, Rothwell JC (2001) The effect on corticospinal volleys of reversing the direction of current induced in the motor cortex by transcranial magnetic stimulation. Exp Brain Res 138:268-273.

Diedrichsen J, Hashambhoy Y, Rane T, Shadmehr R (2005) Neural correlates of reach errors. J Neurosci 25:9919-9931.

Diedrichsen J, White O, Newman D, Lally N (2010) Use-dependent and error-based learning of motor behaviors. J Neurosci 30:5159-5166.

Doyon J, Benali H (2005) Reorganization and plasticity in the adult brain during learning of motor skills. Curr Opin Neurobiol 15:161-167.

Dum RP, Strick PL (2003) An unfolded map of the cerebellar dentate nucleus and its projections to the cerebral cortex. J Neurophysiol 89:634-639.
Fisher KM, Lai HM, Baker MR, Baker SN (2009) Corticospinal activation onfounds cerebellar effects of posterior fossa stimuli. Clin Neurophysiol 120:2019-2213.

Futami T, Kano M, Sento S, Shinoda Y (1986) Synaptic organization of the cerebello-thalamo-cerebral pathway in the cat. III. Cerebellar input to corticofugal neurons destined for different subcortical nuclei in areas 4 and 6. Neurosci Res 3:321-344.

Galea JM, Jayaram G, Ajagbe L, Celnik P (2009) Modulation of cerebellar excitability by polarity-specific noninvasive direct current stimulation. J Neurosci 29:9115-9122.

Ginatempo F, Spampinato DA, Manzo N, Rothwell JC (2019) Exploring the connectivity between the cerebellum and facial motor cortex. Brain Stimul 19:30296-30297.

Hamada M, Strigaro G, Murase N, Sadnicka A, Galea JM, Edwards MJ (2012) Cerebellar modulation of human associative plasticity. J Physiol 590:2365-2374.

Hamada M, Galea JM, Lazzaro V, Mazzone P, Ziemann U, Rothwell JC (2014) Two distinct interneuron circuits in human motor cortex are linked to different subsets of physiological and behavioral plasticity. J Neurosci 34:12837-12849.

Hanajima R, Ugawa Y, Terao Y, Sakai K, Furubayashi T, Machii K, Kanazawa I (1998) Paired-pulse magnetic stimulation of the human motor cortex: differences among I waves. J Physiol 509:607-618.

Hannah R, Rothwell JC (2017) Pulse duration as well as current direction determines the specificity of transcranial magnetic stimulation of motor cortex during contraction. Brain Stimul 10:106-115.

Hardwick RM, Lesage E, Miall RC (2014) Cerebellar transcranial magnetic stimulation: the role of coil geometry and tissue depth. Brain Stimul 7:643-649.

Hashimoto M, Takahara D, Hirata Y, Inoue K, Miyachi S, Nambu A, Tanji J, Takada M, Hoshi E (2010) Motor and non-motor projections from the cerebellum to rostrocaudally distinct sectors of the dorsal premotor cortex in macaques. Eur J Neurosci 31:1402-1413.

Herzfeld DJ, Pastor D, Haith AM, Rossetti Y, Shadmehr R, O'Shea J (2014) Contributions of the cerebellum and the motor cortex to acquisition and retention of motor memories. Neuroimage 98:147-158.

Holdefer RN, Miller LE, Chen LL, Houk JC (2000) Functional connectivity between cerebellum and primary motor cortex in the awake monkey. J Neurophysiol 84:585-590

Ito M (2002) The molecular organization of cerebellar long-term dpression. Nat Rev Neurosci 3:896-902.

Jalali R, Miall RC, Galea JM (2017) No consistent effect of cerebellar transcranial direct current stimulation on visuomotor adaptation. J Neurophysiol 118:655-665.

Jayaram G, Galea JM, Bastian AJ, Celnik P (2011) Human locomotor adaptive learning is proportional to depression of cerebellar excitability. Cereb Cortex 21:1901-1909.

Kelly RM, Strick PL (2003) Cerebellar loops with motor cortex and prefrontal cortex of a nonhuman primate. J Neurosci 23:8432-8444.

Koch G, Porcacchia P, Ponzo V, Carrillo F, Cáceres-Redondo MT, Brusa L (2014) Effects of two weeks of cerebellar theta burst stimulation in cervical dystonia patients. Brain Stimul 7:564-572.

Koziol LF, Budding D, Andreasen N, D'Arrigo S, Bulgheroni S, Imamizu H, Ito M, Manto M, Marvel C, Parker K, Pezzulo G, Ramnani N, Riva D, Schmahmann J, Vandervert L, Yamazaki T (2014) Consensus paper: the cerebellum's role in movement and cognition. Cerebellum 13:151-177.

Marquez CMS, Zhang X, Swinnen SP, Meesen R, Wenderoth N (2013) Taskspecific effect of transcranial direct current stimulation on motor learning. Front Hum Neurosci 7:333

Middleton FA, Strick PL (2000) Basal ganglia and cerebellar loops: motor and cognitive circuits. Brain Res Brain Res Rev 31:236-250.

Na J, Kakei S, Shinoda Y (1997) Cerebellar input to corticothalamic neurons in layers V and VI in the motor cortex. Neurosci Res 28:77-91.

Pinto AD, Chen R (2001) Suppression of the motor cortex by magnetic stimulation of the cerebellum. Exp Brain Res 140:505-510.

Porcacchia P, Álvarez de Toledo P, Rodríguez-Baena A, Martín-Rodríguez JF, Palomar FJ, Vargas-González L, Jesús S, Koch G, Mir P (2019) Abnormal cerebellar connectivity and plasticity in isolated cervical dystonia. PLoS One 14:e0211367.

Reis J, Schambra HM, Cohen LG, Buch ER, Fritsch B, Zarahn E, Celnik PA, Krakauer JW (2009) Noninvasive cortical stimulation enhances motor 
skill acquisition over multiple days through an effect on consolidation. Proc Natl Acad Sci U S A 106:1590-1595.

Rossi S, Hallett M, Rossini PM, Pascual-Leone A (2009) Safety, ethical considerations, and application guidelines for the use of transcranial magnetic stimulation in clinical practice and research. Clin Neurophysiol 120:2008-2039.

Schlerf JE, Galea JM, Spampinato D, Celnik PA (2015) Laterality differences in cerebellar-motor cortex connectivity. Cereb Cortex 25:1827-1834.

Shadmehr R, Smith MA, Krakauer JW (2010) Error correction, sensory prediction, and adaptation in motor control. Annu Rev Neurosci 33:89-108.

Spampinato D, Celnik P (2017) Temporal dynamics of cerebellar and motor cortex physiological processes during motor skill learning. Sci Rep 7:40715.

Spampinato D, Celnik P (2018) Deconstructing skill learning and its physiological mechanisms. Cortex 104:90-102.

Spampinato D, Ibáñez J, Spanoudakis M, Hammond P, Rothwell JC (2020) Cerebellar transcranial magnetic stimulation: the role of coil type from distinct manufacturers. Brain Stimul 13:153-156.

Spampinato DA, Block HJ, Celnik PA (2017) Cerebellar-M1 connectivity changes associated with motor learning are somatotopic specific. J Neurosci 37:2377-2386.

Stefan K, Kunesch E, Cohen LG, Benecke R, Classen J (2000) Induction of plasticity in the human motor cortex by paired associative stimulation. Brain 123:572-584.
Taylor JA, Ivry RB (2014) Cerebellar and prefrontal cortex contributions to adaptation, strategies, and reinforcement learning. Prog Brain Res 210:217-253.

Ugawa Y, Uesaka Y, Terao Y, Hanajima R, Kanazawa I (1995) Magnetic stimulation over the cerebellum in humans. Ann Neurol 37:703-713.

Wagner MJ, Kim TH, Kadmon J, Nguyen ND, Ganguli S, Schnitzer MJ, Luo L (2019) Shared cortex-cerebellum dynamics in the execution and learning of a motor task. Cell 177:669-682.

Werhahn KJ, Taylor J, Ridding M, Meyer BU, Rothwell JC (1996) Effect of transcranial magnetic stimulation over the cerebellum on the excitability of human motor cortex. Electroencephalogr Clin Neurophysiol 101:58-66.

Witham CL, Fisher KM, Edgley SA, Baker SN (2016) Corticospinal inputs to primate motoneurons innervating the forelimb from two divisions of primary motor cortex and area 3a. J Neurosci 36:2605-2616.

Wolpert DM, Diedrichsen J, Flanagan JR (2011) Principles of sensorimotor learning. Nat Rev Neurosci 12:739-751.

Wolters A, Sandbrink F, Schlottmann A, Kunesch E, Stefan K, Cohen LG, Benecke R, Classen J (2003) A temporally asymmetric Hebbian rule governing plasticity in the human motor cortex. J Neurophysiol 89:23392345.

Yamamoto T, Noda T, Miyata M, Nishimura Y (1984) Electrophysiological and morphological studies on thalamic neurons receiving entopedunculoand cerebello-thalamic projections in the cat. Brain Res 301:231-242. 